\title{
Effects of configuration interaction on photoabsorption spectra in the continuum
}

\author{
Yannis Komninos ${ }^{1, *}$ and Cleanthes A. Nicolaides ${ }^{1,2, \dagger}$ \\ ${ }^{1}$ Theoretical and Physical Chemistry Institute, National Hellenic Research Foundation, 48 Vasileos Constantinou Avenue, \\ Athens 11635, Greece \\ ${ }^{2}$ Physics Department, National Technical University, Athens, Greece
}

(Received 15 July 2004; published 22 October 2004)

\begin{abstract}
It is pointed out that the proper interpretation of a recently published experimental spectrum from the multilaser photoionization of Sr [Eichmann et al., Phys. Rev. Lett. 90, 233004 (2003)] must account for a radiative transition between two autoionizing states. The application of orthonormality selection rules and of configuration-interaction theory involving the continuous spectrum and the quasicontinuum of the upper part of Rydberg series explains quantitatively the appearance, the shape, and the variation of heights of the observed peaks of resonances.
\end{abstract}

DOI: 10.1103/PhysRevA.70.042507

PACS number(s): 32.70.-n, 32.30.Jc, 32.80.Rm

\section{INTRODUCTION}

In a recent publication entitled "Fano line shapes reconsidered: Symmetric photoionization peaks from pure continuum excitation," Eichmann, Gallagher, and Konik [1] presented spectroscopic data from a stepwise laser excitation process in $\mathrm{Sr}$ that probed the continuous spectrum below and above the $\mathrm{Sr}^{+} 5 f$ threshold. In this experiment, a $\mathrm{Sr}$ atom is raised via repeated steps of one-photon absorption to the doubly excited $5 d 17 \ell$ state, $\ell=12$, which lies above the energies of the $\mathrm{Sr}^{+} 5 s^{2} S$ and $5 p^{2} P^{o}$ states. From there, a laserinduced photoionization spectrum is recorded, from $\lambda$ $\approx 555$ to $545 \mathrm{~nm}$, thereby sweeping the energy region from slightly below the $\mathrm{Sr}^{+} 5 f$ threshold to the vicinity of the $5 g$ limit at about $544 \mathrm{~nm}$. In this range, there is the continuum of the $\operatorname{Sr} 5 f \varepsilon \ell$ states together with the upper part of the $5 f n \ell$ Rydberg series, as well as the $5 g m \ell^{\prime}$ Rydberg states which, for values of $m \geqslant 20$, lie above the $5 f$ threshold ( $m$ and $n$ are integers, and $\varepsilon$ is the continuous value of the free-electron energy). In other words, the measurements result from a onephoton excitation that starts from the $5 d 17 \ell$ state and reaches the $(5 f n \ell, \varepsilon \ell)$ and $\left(5 g m \ell^{\prime}, \varepsilon^{\prime} \ell^{\prime}\right)$, channels, between which there is configuration interaction (CI). The relevant part of the energy spectrum and the related observations are taken from [1] and are reproduced here for convenience (Figs. 1 and 2).

In order to interpret the observations, Eichmann et al. [1] employed Fano's theory on the "effects of configuration interaction on intensities and phase shifts" [2]. In doing so, they argued that there is a shortcoming in this theory which "is removed by the explicit introduction of the phase-shifted continuum," and proposed modifications of a qualitative nature that account for the upper part of the Rydberg series. Specifically, the data show distinct symmetric peaks, which were attributed to autoionizing states of $5 g m \ell^{\prime}\left(\ell^{\prime}=11,13\right)$ Rydberg configurations, starting with $m=19$, whose energy is just below the $5 f$ threshold, and continuing into the $5 f \varepsilon \ell$

\footnotetext{
*Electronic address: ykomn@eie.gr

${ }^{\dagger}$ Electronic address: can@eie.gr
}

continuum. These configurations cannot be reached by dipole transitions from the $5 d 17 \ell, \ell=12$, state.

In our view, an additional reason for the significance of the published measurements [1] is the fact that the step of photoionization connects two autoionizing states rather than a discrete (initial) and an autoionizing (final) state, which is what is discussed in [1,2]. Given the accuracy of observations, we thought that features of the recorded spectrum should reflect this fact, thereby providing an opportunity to test a previously published related theory and computational methodology [3]. Hence, in the present paper, the observations [1] of symmetric resonances of Sr $5 g m \ell^{\prime}$ configurations below the $\mathrm{Sr}^{+} 5 f$ threshold are interpreted from a different point of view in two ways: first, with respect to the use of electronic structure in conjunction with the theory of configuration interaction in the continuum (CIC) and the incorporation of the upper part of the Rydberg series; second, with respect to the fact that the initial state in the photoionization process, i.e., the $5 d 17 \ell$, is an autoionizing state, since it can mix with the $\operatorname{Sr} 5 s \varepsilon(\ell \pm 2)$ and $5 p \varepsilon(\ell \pm 1)$ continua.

\section{THEORY AND IMPLEMENTATION}

The theory herein is partly based on earlier work on CIC [4-6] which had already pointed out the need for including

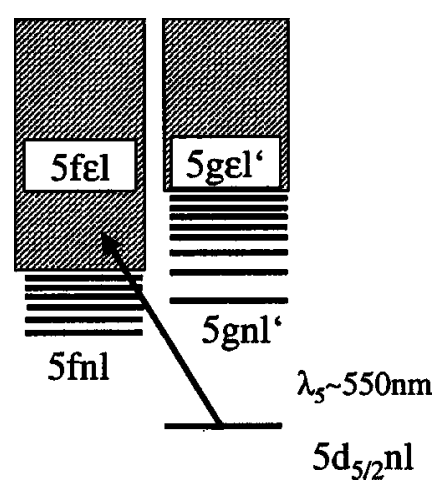

FIG. 1. The energy region probed in the experiment of [1], whose results were analyzed and interpreted in [1] and here. 


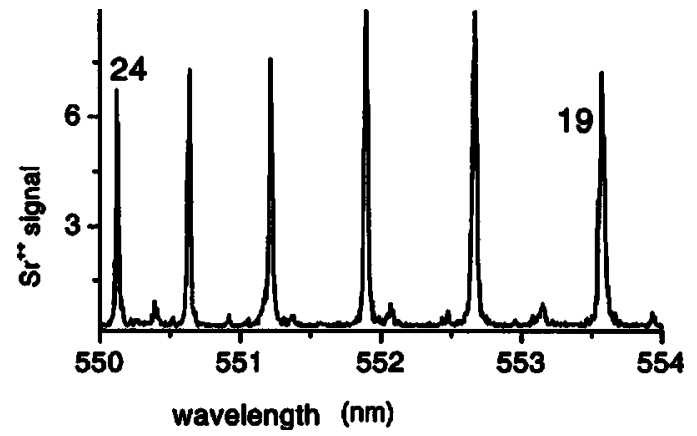

FIG. 2. Detail of the recorded spectrum, taken from Fig. 2b of [1], showing the $5 g m \ell^{\prime}$ resonances, with $m=19-24$.

as an appendage to the Fano formalism the quasicontinuum of Rydberg series below the threshold of interest [4]. The inclusion of such states in a CIC formalism explains the appearance of the symmetric $\mathrm{Sr} 5 g m \ell^{\prime}$ resonances. Use is also made of the work that dealt in a general way with the "theory and computation of the profile of the free-free transition probability between autoionizing states" [3]. Based on this analysis we explain the variation of the heights of the observed peaks, a phenomenon that was not discussed in [1].

In most cases, the determination of the principal features of excited state spectra of polyelectronic atoms or molecules necessitates the superposition of the configurations important for the problem and knowledge of the correct energy differences. Of course, the mixing coefficients result from the complete solution, and one practical question that must concern every formal or computational approach is how to truncate the exact expansion over discrete and scattering basis sets without seriously affecting the correct mixing coefficients of the configurations that determine overwhelmingly the property of interest. The stationary states in the energy range where the symmetric peaks are recorded [1] consist, mainly, of superpositions of the $5 \mathrm{gm}(\ell \pm 1)$ configurations with configurations of the $5 f n \ell, \varepsilon \ell$, channel.

We assume the nonrelativistic Hamiltonian, $H$, with matrix elements $\left\langle 5 g m \ell^{\prime}|H| 5 g m \ell^{\prime}\right\rangle \equiv E_{0}$ and $\left\langle 5 g m \ell^{\prime}|H| 5 f \varepsilon \ell\right\rangle$ $\equiv V_{0 \varepsilon}$, different for each $m$. The intrachannel matrix elements are zero or negligible. In fact, according to analysis of electronic structure, e.g., [7], for the present case of pair correlation with respect to the $5 \mathrm{gm} \ell^{\prime}$ configurations, the angular momentum of the orbitals $\ell$ in the $(5 f n \ell, \varepsilon \ell)$ channel that dominates the interaction matrix elements has the value $\ell=\ell^{\prime}+1$. The main interaction comes from configurations where $n \cong m$. The CI mixing $[5 g m(\ell-1)] \leftrightarrow(5 f n \ell)$ is an example of "symmetric exchange of orbital symmetry" pair correlation [7]. It is due to the increased orbital overlap-and hence interaction - that outer orbitals with $\ell^{\prime}=\ell-1$ have, as compared with those of $\ell^{\prime}=\ell+1$. In what follows we neglect higher-order correlation effects, since they are not relevant to the physics of the discussion.

The observed 5gm $\ell^{\prime}$ Rydberg resonances are well separated from each other [1]. Hence, we opt for the oneresonance formalism which is simpler than the manyresonance one. Following Fano [2], we write the correlated, energy-dependent wave function $\Psi_{E}$ in the vicinity of each $5 \mathrm{gm} \ell^{\prime}$ resonance in the form

$$
\begin{aligned}
\Psi_{E}= & a_{E}\left(\Psi_{0}+\sum_{n} \mathrm{P} \int d E^{\prime} u_{n, E^{\prime}} \frac{1}{E-E^{\prime}} V_{E^{\prime} 0}\right. \\
& \left.+u_{E} \frac{E-E_{0}-\Delta(E)}{V_{E 0}}\right) .
\end{aligned}
$$

The localized part is symbolized by $\Psi_{0}$ (different for each $\left.5 g m \ell^{\prime}\right)$, and has energy $E_{0}$ that is embedded in the continuum of scattering states $5 f \varepsilon \ell$, symbolized by $\left\{\mathrm{u}_{E}\right\}$. P stands for principal value integration. Observe that, next to the scattering states, Eq. (1) contains a summation over the Rydberg states $5 f n \ell$. For energy-normalized wavefunctions, $a_{E}^{2}$ has the form of a Lorentzian distribution with half-width $\Gamma_{E}=\pi V_{0 E}^{2}$. Specifically,

$$
a_{E}^{2}=\frac{1}{\pi} \frac{\Gamma_{E}}{\left[E-E_{0}-\Delta(E)\right]^{2}+\Gamma_{E}^{2}},
$$

where $E>E_{t h}$-the threshold value of $\mathrm{Sr}^{+} 5 f$. The energy shift $\Delta(E)$ is given by

$$
\Delta(E)=\sum_{n} V_{0 E_{n}} \frac{\tau_{n}}{E-E_{n}} V_{E_{n} 0}+\mathrm{P} \int d E^{\prime} V_{0 E^{\prime}} \frac{1}{E-E^{\prime}} V_{E^{\prime} 0}
$$

In the above expression, the matrix elements refer to energynormalized wave functions, and so physical quantities are continued smoothly as the energy crosses the ionization threshold. The correspondence to volume-normalized matrix elements is $V_{0 E_{n}}=\tau_{n}^{-1 / 2} V_{0 n}$ where $\tau_{n}=\partial E_{n} / \partial n$.

CI perturbs the continuum, which is characterized by the phase shift

$$
\delta=-\tan ^{-1}\left(\frac{\Gamma_{E}}{E-E_{0}-\Delta(E)}\right),
$$

while the spectrum of the perturbed Rydberg series results from application of the condition

$$
E_{n}-E_{0}-\Delta\left(E_{n}\right)=0
$$

so that the coefficient of the unbound function $u_{E}$ in Eq. (1) is zero for $E<E_{t h}$.

Equations (3)-(5) show that the existence of a Rydberg series in the escape channel affects the phase shift. This simple extension of Fano's theory was pointed out some time ago [see Eq. (17) of [4] and the general theory of [5,6]], and is basic to the recent discussion by Eichmann et al. [1].

The observation of the symmetric peaks in [1] can be explained using Eq. (1) and elements from the theory of electronic structure [7] in the following way. Since the values of the Rydberg electron angular momentum are high, the states can be treated as single configurations. Adopting the electric dipole approximation, we see that when the $5 d 17 \ell$ configuration is the initial state $\Phi_{\ell}$, the only contribution to the matrix element $\left\langle\Phi_{i}|r| \Psi_{E}\right\rangle$ comes from the Rydberg $5 f 17 \ell$ state, through $\langle 5 d 17 \ell|r| 5 f 17 \ell\rangle \propto\langle 5 d|r| 5 f\rangle\langle 17 \ell \mid 17 \ell\rangle$. Due to electron correlation, $|5 f 17 \ell\rangle$ appears as a component of $\Psi_{E}$ [Eq. (1)] at all energies above and below the $\mathrm{Sr}^{+} 5 f$ threshold. This fact, which results from orthonormality selection rules, is a reasonably general feature of those Rydberg con- 
figurations where the outer orbitals of both initial and final states are essentially hydrogenic.

Therefore, as the energy region with the Rydberg series of autoionizing states $\left|5 \mathrm{gm} \ell^{\prime}\right\rangle$ is reached by one-photon absorption from the $|5 d 17 \ell\rangle$ state, the dipole matrix element for each $\left|5 g m \ell^{\prime}\right\rangle$ of Eq. (1), with $\ell^{\prime}=\ell-1$, is

$$
\left\langle\Phi_{i}|r| \Psi_{E}\right\rangle=a_{E} D_{i E_{17}} \frac{\tau_{17}}{E-E_{17}} V_{E_{17} 0},
$$

where $D_{i E_{17}} \propto\langle 5 d|r| 5 f\rangle\langle 17 \ell \mid 17 \ell\rangle$ and $E_{17}$ is the energy of the $|5 f 17 \ell\rangle$ state. Whenever $E$ is in the neighborhood of the energy of a $\left|5 g m \ell^{\prime}\right\rangle$ Rydberg state with energy $E_{0}+\Delta$, a sharp increase in the cross section takes place because of $a_{E}$ [Eq. (2)].

Hence, there is an absorption peak at the position of each $\mid$ " $5 g m \ell^{\prime}$ " $\rangle$ Rydberg state, with $\ell^{\prime}=\ell-1=11$, whose strength is analogous to the value of $D_{i E_{17}}^{2}$ and to the degree of the contribution of the $|5 f 17 \ell\rangle$ configuration to the wave function (1). The above constitutes a clear interpretation of the measurements of [1] as to the appearance of the peaks inside the continuous spectrum of the $5 f \varepsilon \ell$ channel.

Given Eq. (2), the square of Eq. (6) is a narrow Lorentzian, a fact which explains the observation of symmetric peaks above the $5 f$ threshold. [The quantities $\Gamma$ and $\Delta$, as well as the factor $\left(E-E_{17}\right)^{2}$, are essentially constant.] Below, we show why the Lorentzian shape also holds for the lines due to $\left|5 g m \ell^{\prime}\right\rangle$ states that exist below the $5 f$ threshold. Specifically, we will deduce the similarity which exists between the continuous spectrum and the upper part of the Rydberg series, thereby obtaining an expression [Eq. (11)], for which the form of the distribution is similar to Eq. (2) for the continuum.

The normalization condition of the wave function of Eq. (1) for $E<E_{t h}$ gives

$$
a_{E_{n}}^{2}=\left[1-\frac{d}{d E} \Delta(E)\right]^{-1},
$$

where $E_{n}$ is the energy of each Rydberg state. As written, Eq. (7) cannot be connected to a specific distribution. Nevertheless, the following analysis reveals that $a_{E_{n}}^{2}$ is indeed a Lorentzian.

We first note that the new $\Delta(E)$ has poles at the energies of the zero-order spectrum, the residues of which are all equal to unity. Following [5,6], a smooth function is defined as

$$
\bar{\Delta}\left(E_{\nu}\right)=\Delta\left(E_{\nu}\right)-V_{0 E_{\nu}} \pi \cot \pi \nu V_{E_{\nu} 0},
$$

where the energies of the perturbed spectrum are written as $E_{\nu}=E_{t h}-1 / 2 \nu^{2}$, where $v$ is not an integer (see below). The matrix element $V_{0 E}$ refers to energy-normalized Rydberg states $5 f n \ell$. Then, Eq. (5) takes the form

$$
E_{\nu}-E_{0}-\bar{\Delta}\left(E_{\nu}\right)=V_{0 E_{\nu}} \pi \cot \pi \nu V_{E_{\nu}} 0,
$$

from which $\nu$ is obtained. [Note the similarity with Eq. (4). Putting $\nu=n-\mu$, where $\mu$ is the quantum defect, $\pi \mu$ matches the phase shift $\delta$ at threshold.]
Substituting $\Delta(E)$ of Eq. (3) into Eq. (7), and taking into account the fact that the smooth part is a slowly varying function of the energy, we obtain

$$
\frac{d}{d E} \Delta(E) \approx-\frac{\pi^{2} V_{0 E}^{2}}{\sin ^{2} \pi \nu}\left(\frac{\partial E}{\partial \nu}\right)^{-1}
$$

Putting $\tau_{\nu} \equiv \partial E / \partial \nu=1 / \nu^{3}$, and neglecting unity in Eq. (7), as is natural for high- $n$ Rydberg states, this equation becomes

$$
a_{E_{\nu}}^{2} \approx \frac{\tau_{\nu} \sin ^{2} \pi \nu}{\pi^{2} V_{0 E_{\nu}}^{2}} .
$$

The combination of Eqs. (9) and (11) then gives the Lorentzian shape for a resonance embedded in a quasicontinuum of Rydberg series:

$$
a_{E_{\nu}}^{2} \approx \frac{1}{\pi} \frac{\tau_{\nu} \Gamma_{E_{\nu}}}{\left[E_{\nu}-E_{0}-\bar{\Delta}\left(E_{\nu}\right)\right]^{2}+\Gamma_{E_{\nu}}^{2}} .
$$

This result explains the observed peaks that lie below the $5 f$ threshold, such as the $m=19$ and 18 states [1]. These $\left|5 g m \ell^{\prime}\right\rangle$ states are embedded in the quasicontinuum of the high $|5 f n \ell\rangle$ Rydberg states and indeed their shape is symmetric.

Note that the existence of the factor $\tau_{\nu}$ in Eq. (12) makes possible the evaluation of a sum over the $5 f n \ell$ dense series of states by an integral, according to the recipe $\Sigma_{n} \tau_{n} f_{n}$ $\rightarrow \int d n \tau_{n} f_{n}=\int f(E) d E$. This correspondence is used below, where the third aspect of our discussion, having to do with the radiative transition when the initial state is autoionizing, is presented.

\section{A. Resonance to resonance transition}

Most photoionization experiments in the region of autoionizing (resonance) states involve bound-free transitions. The formalism which explained the phenomenology of the cross section is that of Fano [2].

In our work on free-free transitions [3], we considered the case of radiative transitions between two isolated resonance states. The formalism is general (it includes the Fano case), while the prototypical implementation was concerned with the transition between two doubly excited states of $\mathrm{He}$, " $2 s 2 p "{ }^{1} P^{o} \rightarrow$ " $2 p 3 p$ " ${ }^{1} D$, for tunable radiation around $3.4 \mathrm{eV}$.

Multilaser experiments, such as the one reported in [1], offer the opportunity of measuring accurately the one-photon transition rate between two autoionizing states. In fact, this is what the Sr transition $|5 d 17 \ell\rangle \rightarrow\left|5 g n \ell^{\prime}\right\rangle$ represents, since the initial, stationary state is mixed with the $\operatorname{Sr} 5 s \varepsilon(\ell \pm 2)$ and $5 p \varepsilon(\ell \pm 1)$ continua. Consequently, the initial state of this dipole transition is broadened weakly, with a width $\Gamma_{i}$. The corresponding energy distribution is the Lorentzian

$$
\frac{1}{\pi} \frac{\Gamma_{i}}{\left(E_{i}+h \nu-E\right)^{2}+\Gamma_{i}^{2}},
$$

by which we multiply the square of Eq. (6) and integrate over $E$ to obtain the cross section (apart from constants) [3]. 
The result of the integration of the product of two Lorentzians is a Lorentzian whose width is the sum of the two widths. As a function of $h \nu$, the cross section in the length form is given by [3]

$$
\begin{aligned}
\sigma \propto & \left(E_{m}-E_{i}\right)\left(\frac{\tau_{17}}{E_{i}+h \nu-E_{17}}\right)^{2} \\
& \times \frac{\Gamma_{i}+\Gamma_{m}}{\left[E_{i}+h \nu-E_{m}-\Delta\left(E_{m}\right)\right]^{2}+\left(\Gamma_{i}+\Gamma_{m}\right)^{2}} .
\end{aligned}
$$

This formula is also valid for states lying in the quasicontinuum of the Rydberg series, provided that $\bar{\Delta}$ replaces $\Delta$.

\section{B. Application}

Formula (13) can now be used to interpret the observed heights of the peaks in the spectrum of Sr [1]. Crucial to this is the dependence of the widths on the quantum numbers. Specifically, since both the initial state $5 d 17 \ell$ and the resonance states $5 \operatorname{gm}(\ell-1)$ are of the Rydberg type, their widths are proportional to $\left(17-\mu_{\ell}\right)^{-3}$ and $\left(m-\mu_{0}\right)^{-3}$, respectively, where $\mu$ are quantum defects. At the energies $E_{i}+h \nu=E_{m}$ $+\Delta\left(E_{m}\right), \sigma$ is maximized, with the Lorentzian factor becoming $1 /\left(\Gamma_{i}+\Gamma_{m}\right)$. Therefore, for values $m \gg 17$ the width of the initial state dominates. The result is that the height increases more slowly than it would for a Lorentzian with a width caused by only the $5 \operatorname{gm}(\ell-1)$ state. At the same time, the first factor of formula (13) reduces $\sigma$ with increasing excitation energy. By combining the two results, we find a series of resonances with heights that are slowly decreasing with decreasing wavelength.

The above argument is modified for the $5 \operatorname{gm}(\ell-1)$ states with energies below the $5 f$ threshold (e.g., $m=19$ ), which appear as perturbers of the $5 f n \ell$ Rydberg series, since the sum of the energies in the denominator of Eq. (13) is not equal to zero at the position of the resonance and the Lorentzian does not reach its full height. In this case, this sum is equal to $\Gamma_{m} \cot \pi v$. Using the energies from the Na-

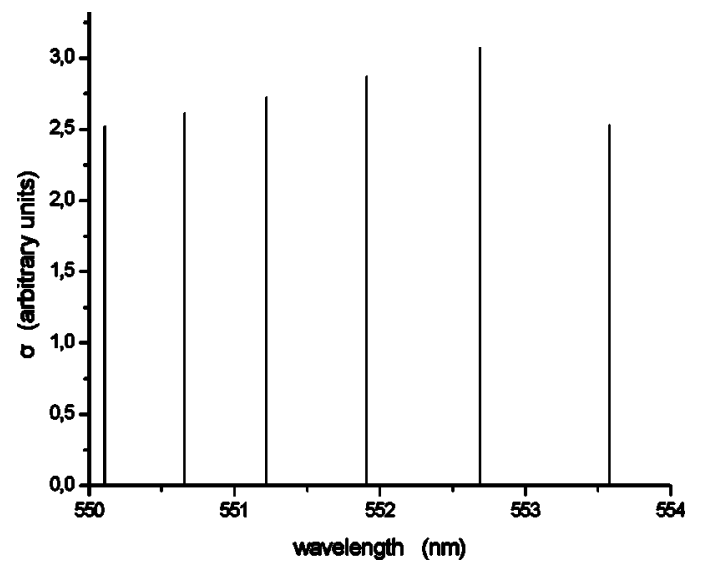

FIG. 3. The line spectrum $\mathrm{Sr} 5 d n \ell \rightarrow 5 f \varepsilon \ell$ (no widths are given), in arbitrary units calculated from formula (13). The positions correspond to the $5 \mathrm{gm} \ell^{\prime}$ resonances, with $m=19-24$.

tional Bureau of Standards tables [8] and the spectrum given in [1], we calculated $\nu=18.8$.

The above theoretical results were applied for the determination of the line spectrum given by Eq. (13) in arbitrary units. The results, Fig. 3, are in excellent agreement with the measurements of [1] (Fig. 2).

\section{CONCLUSION}

The present CIC theory of photoabsorption, which includes as a quasicontinuum the contribution of the upper part of the Rydberg series below the threshold of a channel of physical relevance, explains the observations of Eichmann $e t$ al. [1] in good detail. It is emphasized that the theory is formulated systematically, so as to allow quantitative implementation.

One of the intriguing consequences of CIC occurring in this experiment is the fact that it brings out the fingerprints of dipole transitions between Rydberg resonance states. This is recognized and dealt with here, by treating the problem of resonance-resonance transitions from first principles.
[1] U. Eichmann, T. F. Gallagher, and R. M. Konik, Phys. Rev. Lett. 90, 233004 (2003).

[2] U. Fano, Phys. Rev. 124, 1866 (1961).

[3] Y. Komninos and C. A. Nicolaides, J. Phys. B 30, 811 (1997).

[4] Y. Komninos, N. Makri, and C. A. Nicolaides, Z. Phys. D: At., Mol. Clusters 2, 105 (1986).

[5] Y. Komninos and C. A. Nicolaides, Z. Phys. D: At., Mol. Clusters 4, 301 (1987).

[6] Y. Komninos and C. A. Nicolaides, J. Phys. B 37, 1817 (2004).
[7] C. A. Nicolaides and D. R. Beck, Chem. Phys. Lett. 36, 79 (1975). See articles by D. R. Beck and C. A. Nicolaides, in Excited States in Quantum Chemistry, edited by C. A. Nicolaides and D. R. Beck (Reidel, Dordrecht, 1979).

[8] C. E. Moore, Atomic Energy Levels, Natl. Bur. Stand., U.S. Department of Commerce (U.S. GPO, Washington, D.C., 1971), Vol. II. Note: There is a series of typographical errors in the Table of page 192. The assignments of the $4 p 6(1 S) n g$ configurations should start with $5 g$ at $71357.8 \mathrm{~cm}^{-1}$ and not with $4 g$. 